\title{
A Layout Algorithm for Signal Transduction Pathways as Two-Dimensional Drawings with Spline Curves ${ }^{1}$
}

\author{
Donghoon Lee, Byoung-Hyon Ju, and Kyungsook Han* \\ School of Computer Science and Engineering, Inha University, \\ Inchon 402-751, Korea \\ khan@inha.ac.kr
}

\begin{abstract}
As the volume of the biological pathway data is rapidly expanding, visualization of pathways is becoming an important challenge for analyzing the data. Most of the pathways available in databases are static images that cannot be refined or changed to reflect updated data, but manual layout of pathways is difficult and ineffective. There has been a recent interest in the use of the threedimensional (3D) visualization for signal transduction pathways due to the ubiquity of advanced graphics hardware, the ease of constructing $3 \mathrm{D}$ visualizations, and the common perception of 3D visualization as cutting-edge technology. However, our experience with visualizing signal transduction pathways concluded that $3 \mathrm{D}$ might not be the best solution for signal transduction pathways. This paper presents an algorithm for dynamically visualizing signal transduction pathways as 2D layered digraphs.
\end{abstract}

\section{Introduction}

Recently a number of biological pathway databases have been developed, and visualization of biological networks is crucial to the effective analysis of the data. There are several types of biological networks, such as signal transduction pathways, protein interaction networks, metabolic pathways, and gene regulatory networks. Different types of network represent different biological relationships, and are visualized in different formats in order to convey their biological meaning clearly. The primary focus of this paper is the representation of signal transduction pathways.

A signal transduction pathway is a set of chemical reactions in a cell that occurs when a molecule, such as a hormone, attaches to a receptor on the cell membrane. The pathway is a process by which molecules inside the cell can be altered by molecules on the outside [1]. A large amount of data on signal transduction pathways is available in databases, including diagrams of signal transduction pathways [2, 3, 4]. However, most of these are static images that cannot be changed to reflect updated data. It is increasingly important to visualize signal transduction pathways from databases.

\footnotetext{
${ }^{1}$ This study was supported by the Ministry of Health \& Welfare of Korea under grant 03-PJ1PG3-20700-0040.

* Correspondence Author.
} 
Signal transduction pathways are typically visualized as directed graph (digraph in short) in which a node represents a molecule and an edge between two nodes represents a biological relation between them. Signal transduction pathways convey their meaning best when they are visualized as layered digraphs with uniform edge flows. Therefore, the problem of visualizing signal transduction pathways can be formulated as a graph layout problem. There has been a recent interest in the use of the threedimensional (3D) visualization for signal transduction pathways due to the ubiquity of advanced graphics hardware, the ease of constructing $3 \mathrm{D}$ visualizations, and the common perception of 3D visualization as cutting-edge technology. However, our experience with visualizing signal transduction pathways concluded that 3D might not be the best solution for signal transduction pathways. Because most 3D visualization techniques have a $2 \mathrm{D}$ visualization counterpart, a question arises with respect to the appropriateness of 3D visualization as opposed to $2 \mathrm{D}$ visualization for signal transduction pathways. This paper presents an algorithm for automatically visualizing signal transduction pathways as 2D layered digraphs.

\section{Layout Algorithm}

To discuss the layout algorithm, a few terms should be defined. Suppose that $G=(V$, $E$ ) is an acyclic digraph. A layering of $G$ is a partition of $V$ into subsets $L_{l}, L_{2}, \ldots, L_{h}$, such that if $(u, v) \in E$, where $u \in L_{i}$ and $v \in L_{j}$, then $i>j$. The height of a layered digraph is the number $h$ of layers, and the width of the digraph is the number of nodes in the largest layer. The span of an edge $(u, v)$ with $u \in L_{i}$ and $v \in L_{j}$ is $i-j$.

We visualize signal transduction pathways as layered digraphs. The visualization algorithm is composed of 3 steps at the top level: (1) layer assignment and cycle handling, (2) crossing reduction, and (3) placement of edges with span $>1$.

\subsection{Layer Assignment and Cycle Handling}

This step assigns a y-coordinate to every node by assigning it to a layer. Nodes in the same layer have the same y-coordinate values. It first places all the nodes with no parent in layer $\mathrm{L}_{1}$, and then each remaining node $n$ in layer $L_{p}+1$, where $L_{p}$ is the layer of $n$ 's parent node. When the layer of a node is already determined, the larger value of layers is assigned to the node. Node $\mathrm{L}$ in the middle graph of Fig. 1, for example, is assigned to layer 4 from the path (A, E, I, L), but 3 from the path $(B, G, L)$. The larger value of 4 becomes the layer number of node $\mathrm{L}$.

The main drawback of this layering is that it may produce a too wide digraph and that an edge may have a span greater than one. The number of edges whose span $>1$ should be minimized because they cause the subsequent steps (steps 2-3) of the algorithm take long [9]. We place the source node of an edge whose span $>1$ to higher layers so that the span of the edge becomes one.

Fig. 1 shows an example of the initial layer assignment for the input data of signal transduction below. The initial layer assignment is adjusted to minimize edge spans, as shown in Fig. 2. This step also handles cycles in signal transduction pathways. Starting with a node with no parent node, it assigns a layer to every node along the edges connected to the node. When it encounters a node with a layer assigned to it, it has found a cycle. It starts with a new node no parent node and repeats the same thing. When every node has been assigned a layer, it goes on to step 2. 


$\begin{array}{ll}\text { A } & \text { E } \\ \text { B } & \text { F } \\ \text { B } & \text { G } \\ \text { C } & \text { I } \\ \text { D } & \text { I } \\ \text { E } & \text { I } \\ \text { F } & \text { H } \\ \text { G } & \text { J } \\ \text { G } & \text { L } \\ \text { I } & \text { K } \\ \text { I } & \text { L } \\ \text { K } & \text { D }\end{array}$
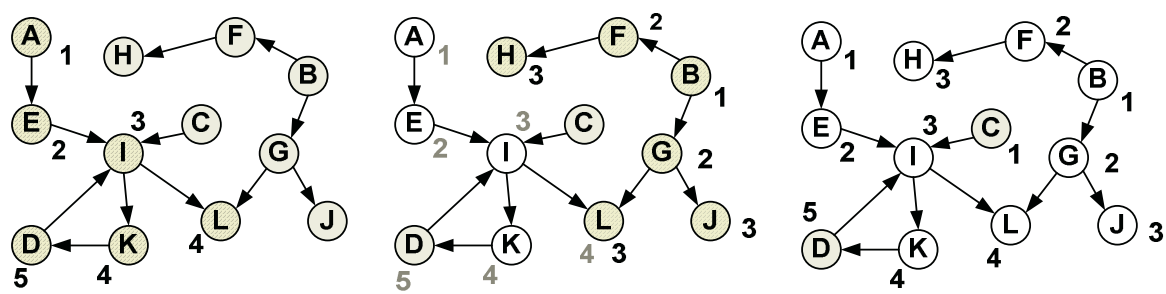

Fig. 1. An example of assigning nodes to layers. The layer numbers in grey indicate the previously assigned numbers. There is a cycle $(\mathrm{D}, \mathrm{I}, \mathrm{K})$ in the graph

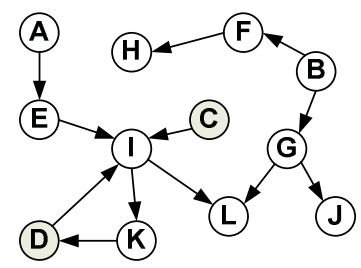

(A)

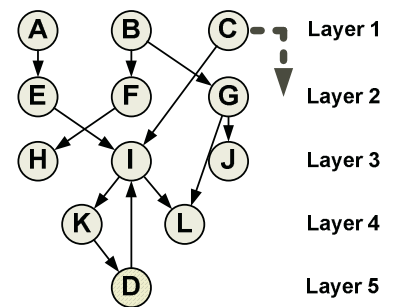

(B)

Fig. 2. (A) Initial digraph. (B) Layered digraph. Node D has an upward edge due to a cycle (I, $\mathrm{K}, \mathrm{D}, \mathrm{I}$ )

Algorithms 1-3 describe step 1 in detail

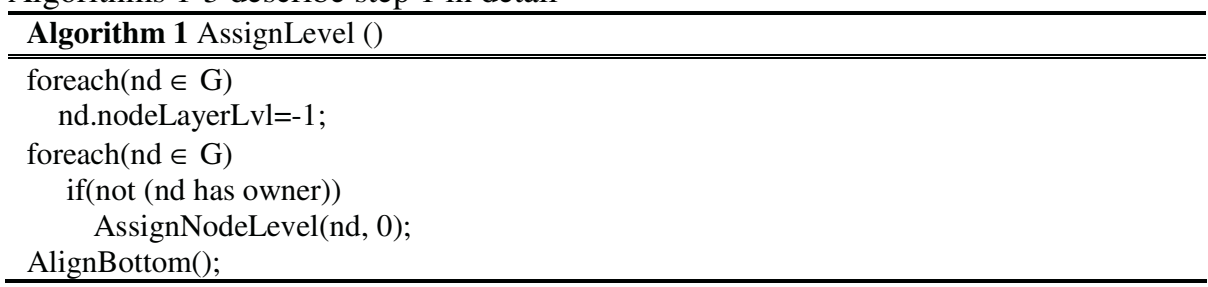



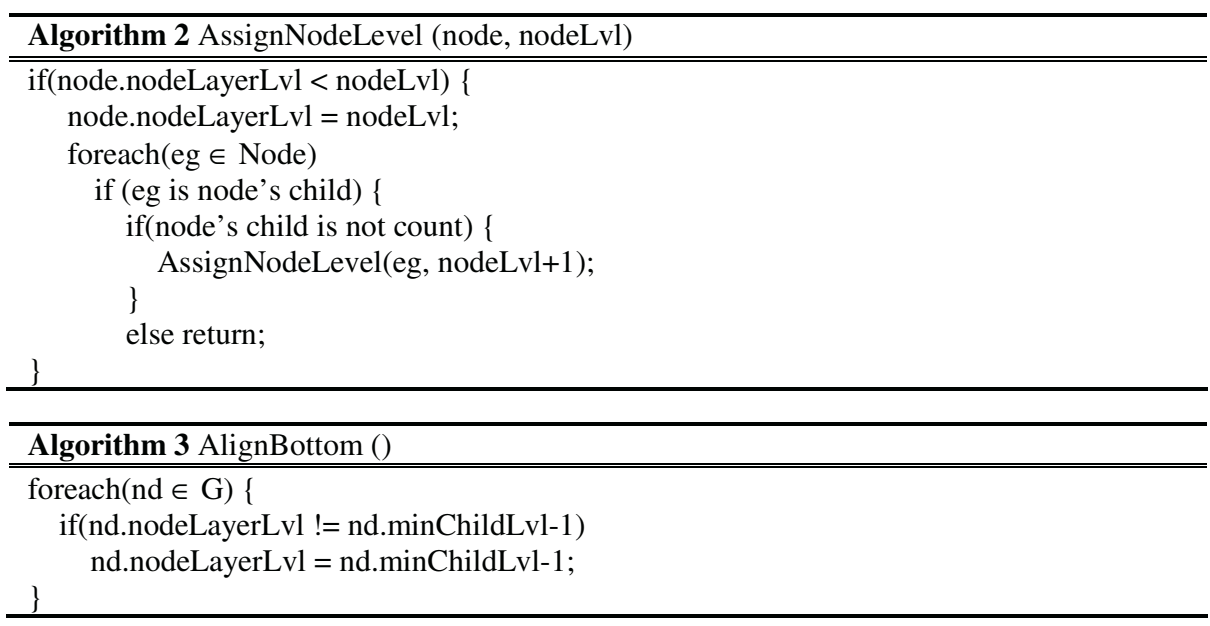

\subsection{Crossing Reduction}

The problem of minimizing edge crossings in a layered digraph is NP-complete, even if there are only two layers [10]. We use the barycenter method to order nodes at each layer $[11,12]$. In the barycenter method, the x-coordinate of each node is chosen as the barycenter (average) of the x-coordinates of its neighbors. Since two adjacent layers are considered in this method, edges whose span $>1$ are ignored in this step.

Suppose that the element $m_{k l}^{(i)}$ of incidence matrix $M(i)$ is given by

$$
m_{k l}^{(i)}=\left\{\begin{array}{lc}
1, & \text { if }\left(v_{k}, v_{l}\right) \in E \\
0, & \text { otherwise }
\end{array}\right.
$$

The row barycenter $\gamma_{k}$ and column barycenter $\rho_{l}$ of incidence matrix $M=\left(m_{k l}\right)$ are defined as

$$
\begin{aligned}
& \gamma_{k}=\sum_{l=1}^{q} l \cdot m_{k l} / \sum_{l=1}^{q} m_{k l} \\
& \rho_{l}=\sum_{k=1}^{p} k \cdot m_{k l} / \sum_{k=1}^{p} m_{k l}
\end{aligned}
$$

The number $\mathrm{C}$ of crossings of the edge between $v_{k}$ and $v_{l}$ is given by

$$
C\left(v_{k}, v_{l}\right)=\sum_{\alpha=k+1}^{p} \sum_{\beta=1}^{l-1} m_{\alpha \beta} \cdot m_{k l}
$$

When rearranging the order of rows (columns), the row (column) barycenters are computed and arranged in increasing order with the order of columns (rows) fixed. By repeatedly alternating row and column barycenter ordering, the total number of crossings is reduced. The algorithm for reducing the total number of crossings is given in Algorithms 5-7, and Fig. 3A shows an example of computing the initial row and col- 
umn barycenters, and the total number of crossings for a graph in Fig. 2B. Fig 3B shows the final row and column barycenters, and the total number of crossings after applying Algorithms 5-7, and the final layout obtained is displayed in Fig. 4A.
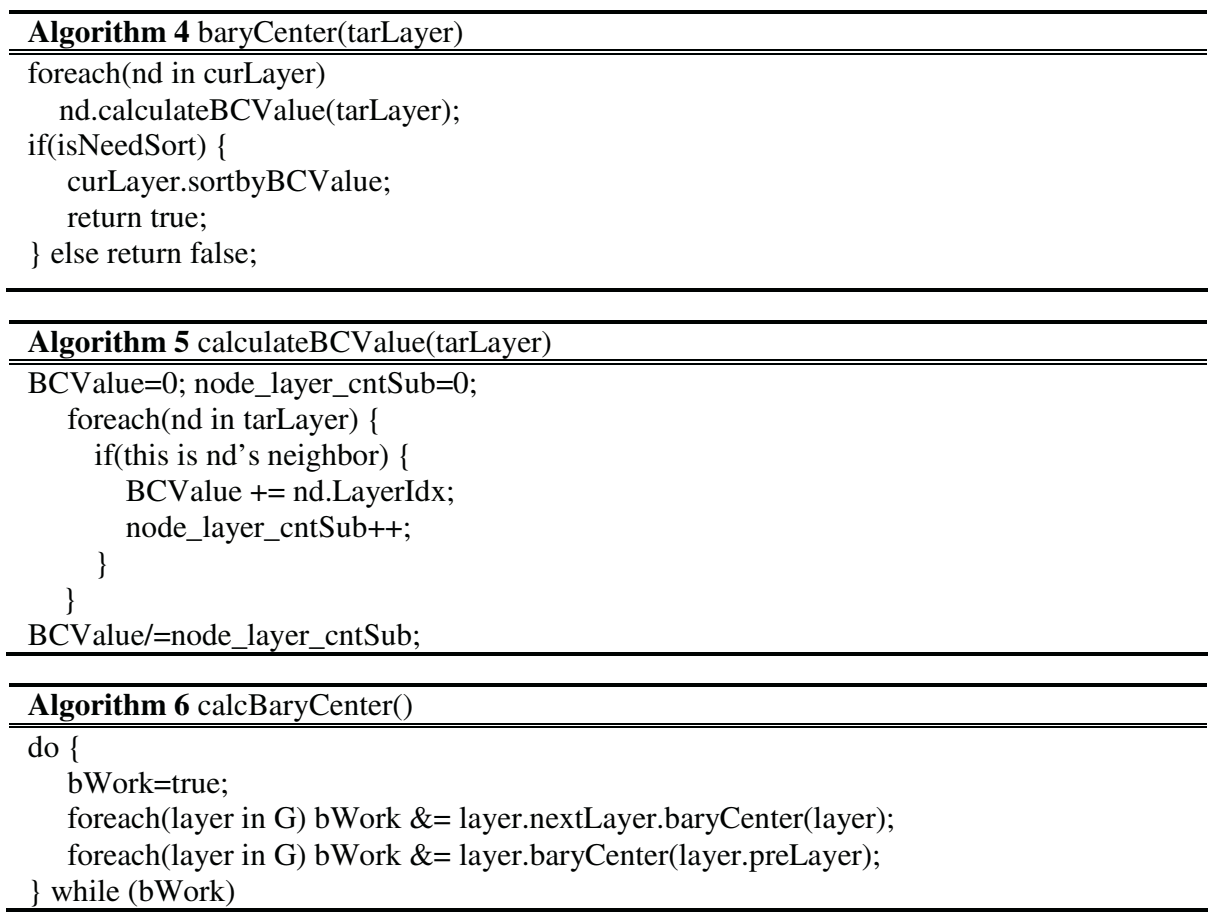

Layer 1 - Layer $2 \quad$ Layer 2 -Layer 3 Layer 3 - Layer $4 \quad$ Layer 4 - Layer 5

(A)

\begin{tabular}{|c|c|c|}
\hline & $C E F G$ & \\
\hline$A$ & 0100 & 2 \\
\hline B & 0011 & 3.5 \\
\hline & 0122 & \\
\hline
\end{tabular}

\section{Crossings $=0$}

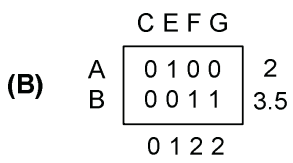

Crossings $=0$

\begin{tabular}{|c|c|c|}
\hline & $\mathrm{HIJ}$ & \\
\hline C & 010 & \\
\hline$E$ & 010 & \\
\hline $\mathrm{F}$ & 100 & \\
\hline $\mathrm{G}$ & 001 & \\
\hline & 31.54 & \\
\hline
\end{tabular}

Crossings $=2$

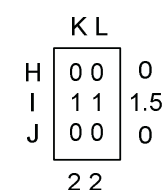

Crossings $=0$

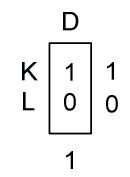

Crossings $=0$

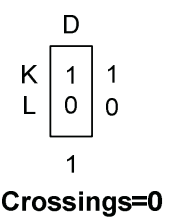

Fig. 3. (A) The initial row and column barycenters, and the total number of crossings for a graph in Fig. 3B. (B) The final row and column barycenters, and the total number of crossings of a graph in Fig. 4A. When rearranging the order of rows and columns based on the row barycenter $\gamma_{k}$ and column barycenter $\rho_{l}$, nodes with $\gamma_{k}=0$ or $\rho_{F}=0$ need not be rearranged 
(A)

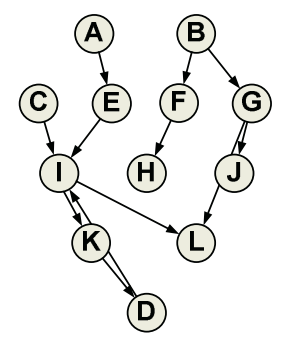

(B)

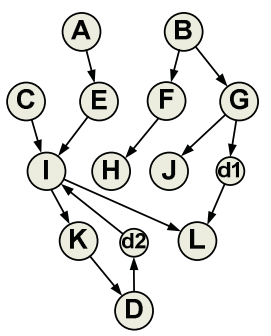

Fig. 4. (A) Digraph with an optimized placement for all nodes and edges except the edges with span $>1$. (B) Graph with dummy nodes introduced for edges with span $>1$. Both nodes $\mathrm{d} 1$ and $\mathrm{d} 2$ are inner dummy nodes

\subsection{Placement of Edges with Span $>1$}

This step places long edges whose edge span $>1$, which have been ignored in the prev ious steps. Every edge with span $=2$ is placed using an inner dummy node. Dummy no des that can be included inside the current graph are called inner dummy nodes, and ar e considered when computing barycenters (Fig. 4B). On the contrary, dummy nodes that cannot be included inside the current graph are called outer dummy nodes and excluded when computing barycenters. For edges with span $>2$, it first computes possible edge $c$ rossings caused by inner dummy nodes and outer dummy nodes and selects whichever with fewer edge crossings. When one or more inner dummy nodes are created, the cross ing reduction step is performed. Otherwise, there is no further crossing reduction.

Inner dummy nodes are created at each layer and the inner dummy nodes are inclu ded when computing barycenters. Therefore, their positions are not fixed until the last step. On the other hand, a pair of outer dummy nodes are created for an edge, one bel ow the source node and the other above the sink node. When the source node is locate $\mathrm{d}$ in the left of the center line of the entire graph, all the outer dummy nodes are place $\mathrm{d}$ in the left of the current graph; otherwise, they are placed in the right side. Edges co nnecting dummy nodes are displayed using spline curves instead of straight line segm ent (see Fig. 6 for an example).

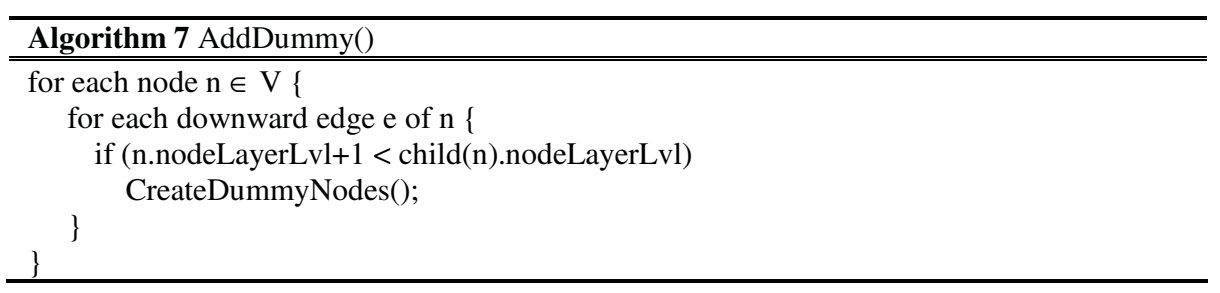

\section{Results}

The algorithms were implemented in a web-based program called PathwayViewer. PathwayViewer runs on Windows 2000/XP/Me/98/NT 4.0 systems. An example of the user interface of the program is shown in Fig. 5. Fig. 6 shows the actual signal 
transduction pathway of mitogen-activated protein kinase. Note the two spline curves for the edges with span $>1$, which have been placed using dummy nodes.

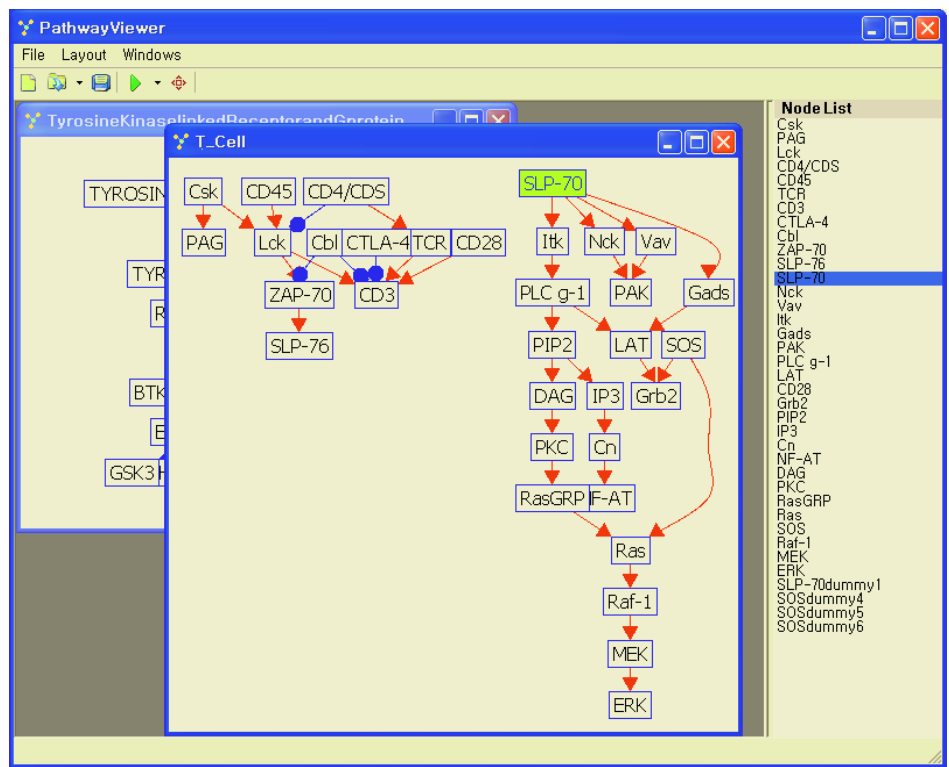

Fig. 5. Example of the user interface of the program. Red arrows indicate the source nodes activate the sink nodes. Blue lines ended with filled circles indicate that source nodes inhibit the sink nodes. The node selected by a user (the yellow node in the signal transduction pathway) is also highlighted in the node list window

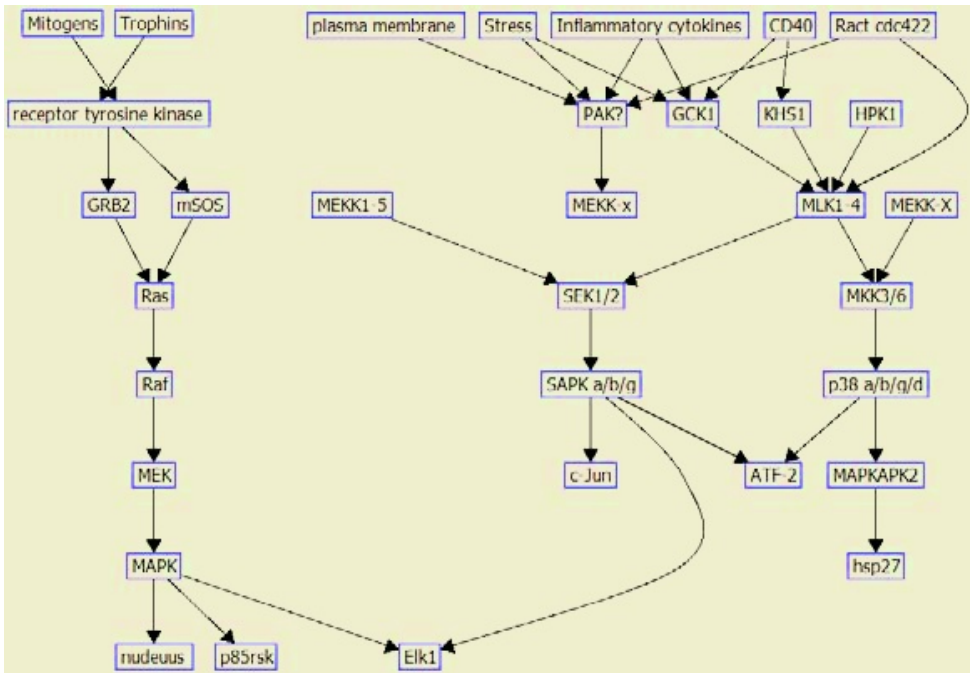

Fig. 6. The signal transduction pathway of mitogen-activated protein kinase, visualized by our algorithm 


\section{Conclusion}

Most databases with signal transduction pathways offer static images. These static images are esthetic since they are hand-made and are fast in loading. However, static images are difficult to refine or change to reflect new data. We have developed an algorithm for automatically representing signal transduction pathways from databases or text files. Unique features of the algorithm include (1) cycles in the pathways are handled; (2) edges with span $>1$ are represented as spline curves; (3) it does not place all sink nodes (nodes with no parent) in layer 1 but moves them to a lower layer so that edge spans can be minimized; and (4) edge bends occur only at dummy nodes and the number of edge bends is minimized. We are currently extending the program to overlay various types of additional information onto the signal transduction pathways.

\section{References}

1. Kanehisa, M., Goto, S., Kawashima, S., Nakaya, A.: The KEGG databases at GenomeNet. Nucleic Acids Research 30 (2002) 42-46

2. Hippron Physiomics, Dynamic Signaling Maps. http://www.hippron.com/products.htm

3. BioCarta._http://www.biocarta.com

4. Kanehisa, M., Goto, S.: KEGG: Kyoto encyclopedia of genes and genomes. Nucleic Acids Research 28 (2002) 27-30

5. Wackett, L., Ellis, L., Speedie, S., Hershberger, C., Knackmuss, H.J., Spormann, A., Walsh, C., Forney, L., Punch, W., Kazic, T., Kaneshia, M., Berndt, D.: Predicting microbial biodegradation pathways. ASM News 65 (1999) 87-93

6. Overbeek, R., Larsen, N., Pusch, G., D’Souza, M., Selkov, E., Kyrpides, N., Fonstein, M., Maltsev, N.: WIT: integrated system for high-throughput genome sequence analysis and metabolic reconstruction. Nucleic Acids Research 28 (2000) 123-125

7. Selkov, E., Grechkin, Y., Mikhailova, N.: MPW: the metabolic pathways database. Nucleic Acids Research 26 (1998) 43-45

8. Karp, P., Riley, M., Saier, M., Paulsen, I., Paley, S. M.: The EcoCyc and MetaCyc databases. Nucleic Acids Research 28 (2000) 56-59

9. Gansner, E.R., Koutsofios, E., North, S.C., Vo, K.-P.: A technique for drawing directed graphs. IEEE Transactions on Software Engineering 19 (1993) 214-230

10. Garey, M.R., Johnson, D.S.: Crossing Number is NP-Complete. SIAM J. Algebraic Discrete Methods 4 (1983) 312-316

11. Sugiyama, K., Tagawa, S., Toda, M.: Method for visual understanding of hierarchical system structures, IEEE Transaction on Systems, Man, and Cybernetics SMC-11 (1981) 109125

12. Sugiyama, K.: Graph Drawing and Applications for Software and Knowledge Engineering. Singapore (2002) 